\title{
Essential Oils from Fresh Fruits, Fruit Stalks and Stem Barks of Four Piper nigrum Varieties from Sarawak
}

\author{
IRNA SYAIRINA SAHARI ${ }^{1}$, ZAINI BIN ASSIM*1, \\ FASIHUDDIN BADRUDDIN AHMAD ${ }^{1} \&$ ISMAIL BIN JUSOH ${ }^{2}$ \\ ${ }^{I}$ Department of Chemistry; ${ }^{2}$ Department of Plant Science and Environmental Ecology, Faculty of \\ Resource Science and Technology, Universiti Malaysia Sarawak, 94300 Kota Samarahan, Sarawak, \\ Malaysia
}

\begin{abstract}
The variation of chemical compositions in essential oils obtained from fresh fruits, fruit stalks and stem barks of four Piper nigrum L. varieties was analysed using capillary gas chromatography-flame ionization detector (GCFID) and gas chromatography-mass spectrometry (GC-MS). The essential oils were extracted by hydrodistillation Clevenger-type apparatus. The major oil constituents identified in all samples were $\beta$-elemene $(1.07-2.93 \%),(+)$ - $\delta$-cadinene $(0.58-6.20 \%), \alpha$-caryophyllene $(1.90-6.21 \%), \delta$-elemene $(0.38-13.26 \%), 3$-carene (0.07-16.82\%) and $\beta$-caryophyllene (11.78-38.33\%). All oil samples showed high content of sesquiterpenes, whereas fruit oils from Semongok Emas and Semongok Wan yielded mostly monoterpenes. Hierarchical clustering and principal component analysis (PCA) of the oil components revealed significant correlation amongst individual $P$. nigrum oil samples.
\end{abstract}

Keywords: P. nigrum, varieties, essential oil, GC-MS, chemometric analysis

\section{INTRODUCTION}

Piper nigrum is a perennial woody climber, which grows up to 10 meter (Ravindran \& Kallupurackal 2000). Its green tinged young stems becoming dark green upon maturity. The fresh unripe fruits are green in color, while ripe fruits are red and dry fruits are black and wrinkled (Barceloux 2009). The dried ground berries are the most widely traded spices in the world. There are various varieties of black pepper and the most popular cultivar in Malaysia is Kuching variety (Ravindran \& Kallupurackal 2000; Janic 2007). Semongok Perak and Semongok Emas varieties of $P$. nigrum were produced from breeding work in the laboratory and were released in 1991 and 1998, respectively (Det 2008). The varieties are known to vary in spike length, berry attributes, quality parameters and yield (Amma et al. 2001). The varieties also differ in resistance to pest and disease (Mammootty et al. 2008). Various investigations focused mainly on the variability of oil compositions between the plant parts. The aims of this study were to

*Corresponding author: zaini@frst.unimas.my evaluate the variation of chemical compositions in the essentials oil of several $P$. nigrum varieties cultivated in Sarawak and classify theme by using chemometric approach.

\section{MATERIALS \& METHODS}

\section{Plant material and hydrodistillation}

Fresh fruits, fruit stalks and stem barks of $P$. nigrum varieties (Kuching, Semongok Emas, Semongok Perak and Semongok Wan) were collected from Tarat, Serian in Sarawak. Identification of $P$. nigrum varieties was performed by Tarat Agricultural Seeding Production Centre. Plant materials (100 g) were subjected to hydrodistillation in a Clevengertype apparatus for 8 hours. The oils collected were dried over sodium sulphate anhydrous. The oil yield was calculated and recorded on the basis of dried weight material.

\section{GC-FID and GC-MS analysis}

GC-FID analysis on the essential oils constituents were performed by using a Hewlett Packard HP-6890 gas chromatograph equipped 
with a flame ionization detector (FID) using a HP-5 fused capillary column ( $30 \mathrm{~m}$ x $0.25 \mathrm{~mm}$ i.d. $\mathrm{x} 0.25 \mu \mathrm{m}$ of phase thickness). Hydrogen was used as the carrier gas at a flow rate of 1 $\mathrm{mL} / \mathrm{min}$. Exactly $1 \mu \mathrm{L}$ of diluted sample was injected in splitless mode. The oven temperature was initially set at $40{ }^{\circ} \mathrm{C}$ for $3 \mathrm{~min}$, and then programmed at rate of $8{ }^{\circ} \mathrm{C} / \mathrm{min}$ to $160{ }^{\circ} \mathrm{C}$ and at a rate of $15{ }^{\circ} \mathrm{C} / \mathrm{min}$ to $300{ }^{\circ} \mathrm{C}$. The final temperature was held constant for 10 $\min$.

GC-MS analysis of essential oil was performed on a GC-MS model Shimadzu QP2010 Plus. Prior to GC-MS analysis, exactly 1 $\mu l$ of diluted essential oil was injected into the column in the splitless mode. The essential oil sample was separated on a capillary BPX-5 column $(30 \mathrm{~m}$ x $0.25 \mathrm{~mm}$ i.d. $\times 0.25 \mu \mathrm{m}$ of film thickness). The temperature program used was similar to GC-FID analysis. The injector temperature was set at $25^{\circ} \mathrm{C}$. Helium gas was used as carrier gas with a flow rate of 1 $\mathrm{mL} / \mathrm{min}$.

Identification of the individual components was made by comparison of their mass spectral with the National Institute for Standard Technology - NIST08 library incorporated in GC-MS data system and reconfirmed by comparing the calculated Kovat's indices with Liu et al. (2011). The Kovats index was calculated using n-alkanes homologous series. The semi-quantitative data of the oils were obtained using peak area of each component in the gas chromatogram without applying correction factors.

\section{Chemometric statistical analysis}

Prior to cluster analysis (CA) and principal component analysis (PCA), the set of data was processed using PASW Statistics 18 software. A combination of CA and PCA were used to assess variability existing in composition of essential oils from different varieties of $P$. nigrum.

\section{RESULTS \& DISCUSSION}

\section{The Percentage Yield of the Essential Oils}

The oil yield of samples calculated on dry weight basis showed that the fruit stalk gave highest yield $(2.49-3.80 \%$, v/w), while stem bark showed the lowest yield of oil (0.33 $1.13 \%, \mathrm{v} / \mathrm{w})$. The fruit gave oil yield between $1.05-1.49 \%(\mathrm{v} / \mathrm{w})$ and all the oil was in liquid form. The physical properties of each oil types are summarized in Table 1.

\section{Chemical Composition of the Essential Oils}

Figure 1 shows a typical GC-MS chromatogram of essential oils from P. nigrum. The identified oil constituents and their Kovat's indices are listed in Table 2. The major components identified in the $P$. nigrum oil were monoterpene (3-carene) and sesquiterpenes $(\beta$ elemene, $(+)$ - $\delta$-cadinene, $\alpha$-caryophyllene, $\delta$ -

Table 1. Percentage yield and physical properties of oil isolated from P. nigrum varieties.

\begin{tabular}{llcc}
\hline Plant part & Variety & $\begin{array}{c}\text { Oil yield } \\
(\% \mathrm{v} / \mathrm{w})\end{array}$ & Oil colour \\
\hline Fruit & Kuching & 1.43 & Light yellow \\
& Semongok Emas & 1.05 & Light yellow \\
& Semongok Perak & 1.34 & Light yellow \\
Fruit stalk & Semongok Wan & 1.49 & Light yellow \\
& Kuching & 3.80 & Light greenish yellow \\
& Semongok Emas & 3.07 & Light yellow \\
\multirow{5}{*}{ Stem bark } & Semongok Perak & 2.49 & Dark yellow \\
& Semongok Wan & 3.77 & Light yellow \\
& Kuching & 0.88 & Light yellow \\
& Semongok Emas & 1.04 & Light yellow \\
& Semongok Perak & 0.33 & Light yellow \\
& Semongok Wan & 1.13 & Light yellow \\
\hline
\end{tabular}


elemene and $\beta$ - caryophyllene). A total of 19 monoterpenes, 78 sesquiterpenes and 3 nonterpenes compounds were identified in the oil samples. Monoterpenes were detected in the range of $0.07-55.50 \%$ in oils from different varieties. The presence of monoterpenes in fruit oils were significantly high $(31.57-55.50 \%)$ but very low in stem bark oils $(0.07-8.39 \%)$. Monoterpenes were dominated by hydrocarbon monoterpenes, such as $\alpha$-pinene $(0.56-$ $4.58 \%), \beta$-pinene $(1.86-7.97 \%)$ and limonene $(3.24-14.47 \%)$. 3-Carene was the most abundant monoterpene $(0.07-16.82 \%)$ identified. This finding is different from previous studies (Menon et al. 2003; Menon \& Padmakumari 2005a) which reported $\beta$-pinene as the main constituents in the fruit oils of $P$. nigrum. High content of sabinene was reported in the fruit oil of P. nigrum (Menon et al. 2003; Tchoumbougnang et al. 2009) but sabinene was identified only in fruits oil of Kuching and Semongok Emas varieties. The fruits oil from Semongok Emas has the highest percentage of hydrocarbon monoterpene (53.78\%) among varieties, while the stem bark oil-from Semongok Wan showed the lowest content (0.07\%). Menon et al. (2005b) reported $\alpha$ pinene $(2.3-5.4 \%)$ as the principal monoterpene in fruits oil of Kuching variety and similar result was observed in fruits oil where Kuching variety contained $3.33 \%$ of $\alpha$-pinene. Only a few oxygenated monoterpenes were identified and Semongok Emas fruits oil recorded the highest content (1.71\%). Oxygenated monoterpenes was not detected in stem bark oil of Kuching and Semongok Wan varieties.

Approximately $44.50-98.87 \%$ of total content of P. Nigrum oil samples were sesquiterpenes, mainly in fruit stalk oils (87.31$90.45 \%$ ) and stem bark oils (78.01-98.87\%). The stem barkoils reported the highest percentage of hydrocarbon sesquiterpenes (36.68-82.84\%), while the fruit oils gave the lowest percentage of hydrocarbon sesquiterpenes (39.68-59.10\%).The major hydrocarbon sesquiterpene identified in all oil samples was $\beta$-caryophyllene (11.78-38.33\%). This finding is similar with those previous reports (Pino et al. 2003; Martins et al. 1998; Sasidharan \& Menon 2010). Pino et al. (2003) have identified $\alpha$-terpinene with significant amount in stem bark oil but less than $1 \%$ of this compound was detected from the stem bark oil in this study. Hydrocarbon sesquiterpene were abundance in fruit stalk oil of Semongok Perak variety $(81.54 \%)$, while the fruit oil of Semongok Emas variety contained only $39.68 \%$ of hydrocarbon sesquiterpene. Oxygenated sesquiterpenes represent 1.38$16.74 \%$ of total oil content. The most abundance oxygenated hydrocarbon compounds identified are caryophyllene oxide $(0.30-3.06 \%)$ and spathulenol (0.06-5.49\%). Oxygenated sesquiterpenes were found mostly in the stem bark oil of Kuching variety $(16.74 \%)$. Only a few of oxygenated sesquiterpenes were identified in fruit oil of Semongok Perak variety (1.38\%).

\section{Statistical Analysis on Essential Oil Constituents}

The chemometric analysis of oil components revealed similarity between plant parts among $P$. nigrum varieties. In comparison of similarity level of plant parts from $P$. nigrum varieties, some close relationships were revealed, especially among the fruit oils. Dendrogram

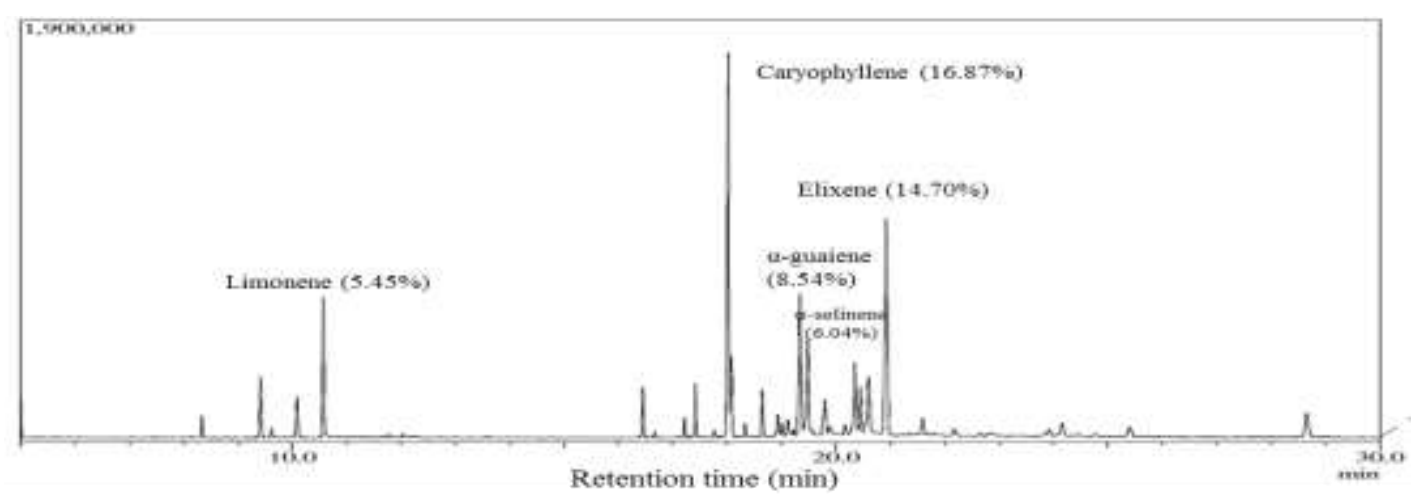

Figure 1. A GC chromatogram of fruit stalk oil from Kuching variety of P. nigrum. 
(Figure 2) and PCA plot (Figure 3) showed that the fruit oil of Semongok Perak variety has the highest similarity level with that of Semongok Wan variety fruit oils.

The fruit oil of Semongok Perak variety also has some similarity with the fruit oil of Semongok Emas variety. There were also some correlations between the stem bark oils in all samples. The stem bark oil of Kuching variety was clustered next to stem bark oil Semongok Perak variety. The stem bark oil of Kuching variety was also correlated to Semongok Emas variety stem bark oil. There was some similarity between the fruit stalk oils of Kuching and Semongok Perak varieties.

Results obtained from cluster analysis and principal component analysis also exhibited the existence of several variations within the oils according to the plant parts. The stem bark and fruit stalk oils of Semongok Wan were clustered separately from other oil samples as shown in Figures 2 and 3.

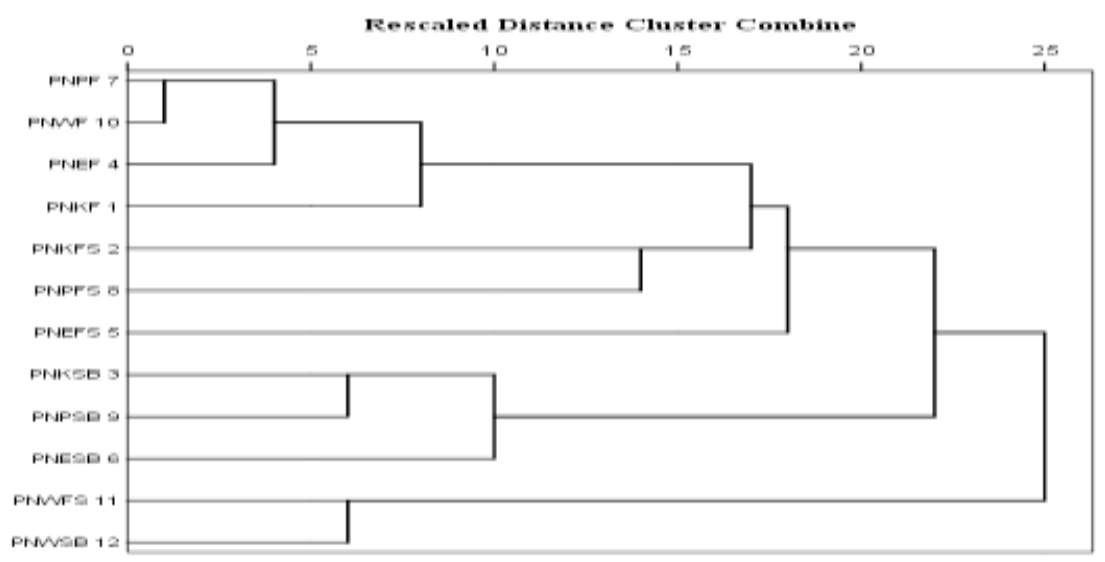

Figure 2. Two-dimensional dendrogram obtained in the hierarchal cluster analysis of fruits, fruit stalks and stem barks oils from four $P$. nigrum varieties based on the GC-MS analysis of essential oils. Vertical: Oil samples analysed; Horizontal: Similarity level between oil samples. Distance cluster is the distance of each oil sample from all the others.

Note: PNKF: Kuching variety (fruit); PNKFS: Kuching variety (fruit stalk); PNKSB: Kuching variety (stem bark); PNEF: Semongok Emas variety (fruit); PNEFS: Semongok Emas variety (fruit stalk); PNESB: Semongok Emas variety (stem bark); PNPF: Semongok Perak variety (fruit); PNPFS: Semongok Perak variety (fruit stalk); PNPSB: Semongok Perak variety (stem bark); PNWF: Semongok Wan variety (fruit); PNWFS: Semongok Wan variety (fruit stalk); PNWSB: Semongok Wan variety (stem bark)

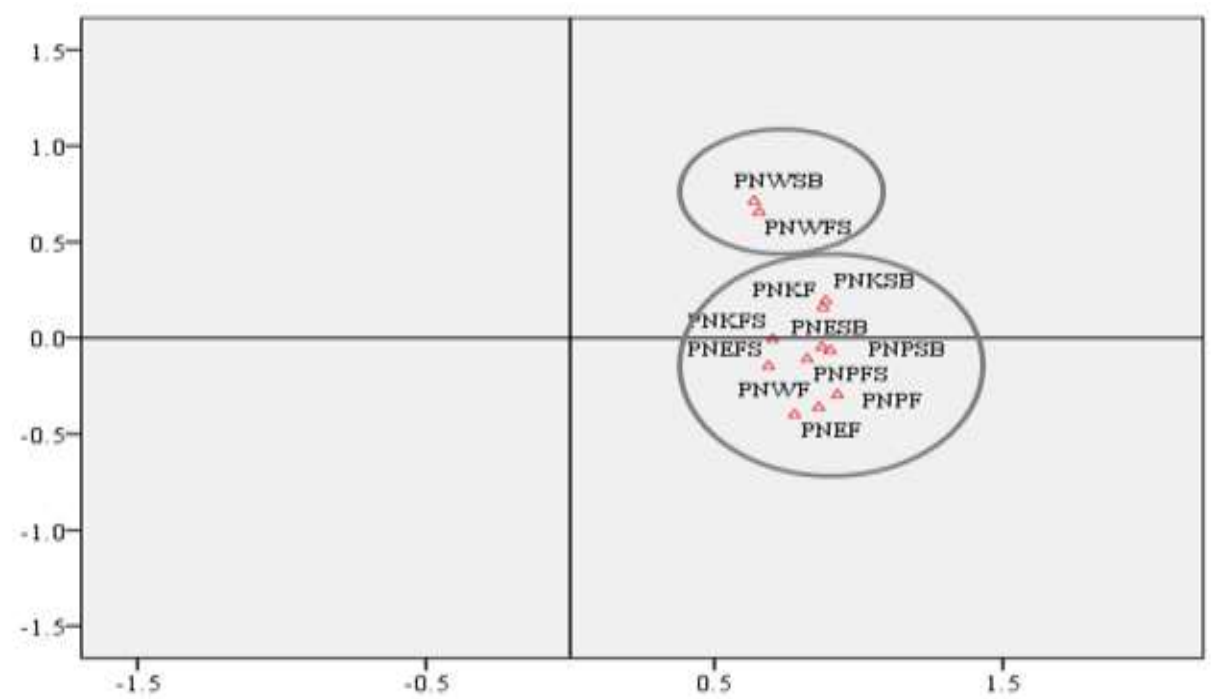

Figure 3. Component plot obtained in PCA of fruit, fruits stalk and stem bark of four P. nigrum varieties based on their oil constituents identified by GC-MS

Note: PNKF: Kuching variety (fruit); PNKFS: Kuching variety (fruit stalk); PNKSB: Kuching variety (stem bark); PNEF: Semongok Emas variety (fruit); PNEFS: Semongok Emas variety (fruit stalk); PNESB: Semongok Emas variety (stem bark); PNPF: Semongok Perak variety (fruit); PNPFS: Semongok Perak variety (fruit stalk); PNPSB: Semongok Perak variety (stem bark); PNWF: Semongok Wan variety (fruit); PNWFS: Semongok Wan variety (fruit stalk); PNWSB: Semongok Wan variety (stem bark) 
Table 2. Chemical compounds identified in fruit, fruit stalk and stem bark oils for P. nigrum samples

\begin{tabular}{|c|c|c|c|c|c|c|c|c|c|c|c|c|}
\hline \multirow[b]{2}{*}{ Chemical Composition (KI*) } & \multicolumn{12}{|c|}{ Percentage of compound (\%) } \\
\hline & $\mathrm{KF}$ & KFS & KSB & $\mathrm{EF}$ & EFS & ESB & $\mathrm{PF}$ & PFS & PSB & WF & WFS & WSB \\
\hline \multicolumn{13}{|l|}{ Monoterpene hydrocarbons } \\
\hline$\alpha$-Thujene (931) & 0.38 & - & - & 1.05 & 0.09 & - & - & - & - & - & 0.31 & - \\
\hline$\alpha$-Pinene (938) & 3.33 & 0.80 & - & 4.58 & 1.12 & 0.28 & 4.13 & 0.56 & 0.30 & 3.88 & 0.78 & - \\
\hline Sabinene (979) & 2.82 & - & - & 6.41 & - & 0.50 & - & - & - & - & - & - \\
\hline$\beta$-Pinene (985) & 5.80 & 2.46 & 0.10 & 6.08 & 1.87 & 0.23 & 6.93 & 1.86 & 0.38 & 7.97 & 1.76 & - \\
\hline$\beta$-Myrcene (993) & 1.34 & 0.41 & - & 2.18 & 0.37 & 0.18 & 1.75 & 0.38 & 0.12 & 2.37 & 0.30 & - \\
\hline 3-Carene (1014) & 6.18 & 1.80 & 0.58 & 16.82 & 3.94 & 5.17 & 12.31 & 2.46 & 3.83 & 16.11 & 0.96 & 0.07 \\
\hline$\alpha$-Terpinene (1024) & - & - & - & 0.27 & 0.13 & 0.05 & 0.50 & - & - & 0.28 & 0.10 & - \\
\hline Limonene (1035) & 9.83 & 5.45 & 0.29 & 10.36 & 3.94 & 0.90 & 11.65 & 5.33 & 0.94 & 14.47 & 3.24 & - \\
\hline$\beta$-Phellandrene (1040) & 1.22 & - & - & 2.79 & 0.24 & 0.35 & 0.59 & 0.07 & 0.10 & 1.04 & 0.16 & - \\
\hline$\delta$-Terpinene (1067) & - & - & - & 0.77 & - & 0.18 & - & - & - & 0.38 & - & - \\
\hline Terpinolene (1089) & 0.38 & 0.20 & - & 1.41 & 0.27 & 0.12 & 0.87 & 0.15 & - & 1.46 & 0.15 & - \\
\hline Other monoterpene hydrocarbons & & & & 1.06 & 0.16 & 0.55 & & & & 1.36 & 0.61 & \\
\hline Sub-total & 31.29 & 11.13 & 0.97 & 53.78 & 12.13 & 8.51 & 38.98 & 11.38 & 5.83 & 49.32 & 8.36 & 0.07 \\
\hline \multicolumn{13}{|l|}{ Oxygenated monoterpene derivatives } \\
\hline 6-methyl-2-(oxiran-2-yl)hept-5-en-2-ol (1080) & - & & - & - & 0.17 & - & - & - & - & - & - & - \\
\hline Linalool (1107) & 0.28 & 0.19 & - & 0.90 & 0.24 & 0.07 & 0.54 & 0.33 & 0.62 & 1.54 & 0.42 & - \\
\hline 1-Terpinen-4-ol (1196) & - & & - & 0.82 & - & 0.07 & - & - & - & - & 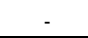 & - \\
\hline Sub-total & 0.28 & 0.19 & 0.00 & 1.71 & 0.41 & 0.13 & 0.54 & $\mathbf{0 . 3 3}$ & 0.62 & 1.54 & 0.42 & 0.00 \\
\hline \multicolumn{13}{|l|}{ Sesquiterpene hydrocarbons } \\
\hline$\delta$-Eiemene $(1345)$ & 2.88 & 1.88 & 0.38 & 5.23 & 1.62 & 0.86 & 8.95 & 13.26 & 7.09 & 4.66 & 3.44 & 3.00 \\
\hline Copaene (1390) & 0.29 & 0.75 & 0.13 & 3.53 & 7.39 & 5.27 & 6.89 & 8.90 & 3.03 & 5.40 & - & 0.22 \\
\hline$\beta$-Elemene (1401) & 1.44 & 2.09 & 1.87 & 1.07 & 2.42 & 2.07 & 2.50 & 2.93 & 2.38 & 2.17 & 1.89 & 3.04 \\
\hline$(+)-\alpha$-Longipinene $(1421)$ & - & 1.11 & - & - & 0.67 & 0.44 & - & 0.40 & - & - & 1.12 & 0.43 \\
\hline Cedr-8-ene (1423) & - & 0.40 & - & - & 0.86 & - & - & 1.19 & - & - & 0.52 & - \\
\hline$\alpha$-Bergamotene (1423) & 0.31 & 0.89 & 0.25 & 0.25 & 0.65 & - & 0.49 & 0.15 & - & 0.55 & 1.42 & - \\
\hline$\beta$-Caryophyllene (1437) & 18.90 & 16.87 & 38.33 & 15.29 & 15.64 & 36.86 & 25.87 & 21.73 & 33.20 & 21.82 & 11.78 & 16.82 \\
\hline$\gamma$-Elemene $(1440)$ & 1.77 & 3.17 & 1.71 & 0.29 & 1.52 & - & - & 0.53 & - & 0.28 & 3.72 & 4.73 \\
\hline Germacrene D (1446) & 2.77 & 0.13 & 0.32 & 4.33 & 0.19 & 16.55 & 0.77 & 0.29 & 0.22 & 0.61 & - & 2.89 \\
\hline$\beta$-Cubebene (1447) & - & - & - & - & 0.59 & - & - & - & - & - & 0.08 & 0.16 \\
\hline (Z)- $\beta$-Farnesene (1455) & 0.76 & 0.73 & 1.16 & 0.28 & 0.74 & 0.33 & - & 1.13 & - & - & 1.03 & 0.65 \\
\hline$\alpha$-Caryophyllene (1473) & 2.45 & 2.13 & 4.43 & 1.90 & 1.93 & 4.21 & 3.39 & 3.74 & 6.21 & 2.97 & 2.23 & 2.32 \\
\hline Isoledene (1479) & - & 1.14 & - & - & 17.08 & 0.21 & - & 2.42 & 1.90 & - & 2.13 & 0.15 \\
\hline$\beta$-Muurolene (1490) & - & 1.29 & 1.05 & - & 1.60 & 1.36 & - & 0.57 & 0.37 & - & 1.30 & 1.28 \\
\hline Cycloisosativene (1494) & - & 0.10 & 0.36 & - & 1.42 & 1.39 & - & 0.20 & 3.82 & - & 0.22 & 0.89 \\
\hline$\alpha$-Zingiberene (1505) & 0.49 & 0.50 & 0.52 & 0.23 & 0.75 & - & - & 0.06 & - & - & 1.35 & 0.87 \\
\hline$\beta$-Selinene (1509) & - & & - & - & - & - & - & - & 6.04 & - & & - \\
\hline$\alpha$-Guaiene (1511) & - & 8.54 & - & - & 1.11 & - & 2.27 & 6.12 & - & - & 7.03 & 0.34 \\
\hline Elixene (1513) & 0.36 & 14.70 & - & - & - & - & - & 0.28 & 0.37 & 3.12 & 0.61 & - \\
\hline$\alpha$-Amorphene (1513) & - & - & - & - & 1.80 & 0.54 & - & - & - & - & - & 0.55 \\
\hline$\beta$-bisabolene (1516) & 6.22 & - & 6.95 & 3.20 & 7.66 & & - & - & - & - & 5.57 & \\
\hline
\end{tabular}




\begin{tabular}{|c|c|c|c|c|c|c|c|c|c|c|c|c|}
\hline$\alpha$-Selinene (1517) & & 6.04 & - & & - & - & 2.36 & 6.08 & 3.76 & - & & - \\
\hline$\delta$-Cadinene (1530) & 0.58 & 2.82 & 2.27 & 2.36 & 6.20 & 4.52 & 3.82 & 4.88 & 2.52 & 2.96 & 2.15 & 2.62 \\
\hline$\alpha$-Farnesene (1534) & 0.92 & - & - & 0.38 & - & - & - & - & - & - & - & - \\
\hline$\alpha$-Patchoulene(1534) & - & - & 0.84 & - & 1.19 & - & - & - & - & - & 0.28 & 1.56 \\
\hline cis- $\alpha$-Bisabolene (1546) & 0.74 & - & - & - & - & - & 0.21 & - & - & - & - & - \\
\hline$\alpha$-Gurjunene (1546) & - & 0.68 & 1.04 & - & 0.51 & - & - & 3.39 & 0.81 & - & 2.39 & 1.30 \\
\hline$\alpha$-Muurolene (1551) & - & - & - & - & 0.41 & 0.32 & 0.52 & - & 0.11 & - & - & - \\
\hline $\begin{array}{l}\text { 1,1,3a-trimethyl-7- methylene decahydro-1H- } \\
\text { cyclopropa[a]- naphthalene (1554) }\end{array}$ & - & - & 7.83 & - & - & - & - & - & 0.20 & & - & 3.64 \\
\hline Valencene (1555) & . & 4.96 & $1.0 J$ & - & - & - & - & - & -2.20 & - & 4.85 & $\begin{array}{c}3.04 \\
-\end{array}$ \\
\hline Eudesma-3,7(11)-diene (1559) & - & 3.10 & 4.95 & - & 0.23 & - & . & 0.07 & - & - & 3.03 & - \\
\hline Germacrene B (1579) & 10.68 & - & 7.61 & 0.29 & - & 1.21 & . & 0.04 & - & - & 17.11 & 25.59 \\
\hline$\beta$-Cadinene (1658) & - & - & - & - & 0.29 & 0.20 & 0.34 & 1.00 & 0.92 & - & 0.33 & - \\
\hline$\gamma$-Gurjunene (1679) & - & 0.36 & - & - & 0.39 & 0.21 & - & 0.53 & 0.72 & - & 0.26 & 0.28 \\
\hline Other Sesquiterpene hydrocarbons & 0.75 & 0.19 & & 0.28 & 0.83 & 0.87 & & 1.10 & 4.62 & & 0.37 & 0.41 \\
\hline Sub-total & 52.29 & 74.84 & 82.12 & 39.68 & 77.86 & 79.42 & 59.10 & 82.84 & 78.81 & 45.12 & 76.40 & 73.91 \\
\hline \multicolumn{13}{|l|}{ Oxygenated sesquiterpenes derivatives } \\
\hline Isofuranogermacren e (1510) & 5.28 & 1.23 & 5.75 & - & - & - & - & - & - & 2.46 & 0.68 & - \\
\hline$\alpha$-trans-Bergamotol (1519) & 0.63 & - & - & - & - & - & - & - & - & - & 0.56 & 0.58 \\
\hline Elemol (1562) & 0.40 & & - & - & 0.28 & - & - & - & - & - & - & - \\
\hline trans-Nerolidol (1565) & 3.49 & 4.69 & 7.59 & - & 0.21 & - & - & - & - & 0.63 & 4.13 & - \\
\hline Germacrene D-4-ol (1599) & & 0.36 & - & - & 0.22 & - & . & 0.24 & - & - & 0.27 & 0.10 \\
\hline Caryophyllene oxide (1606) & 2.49 & 1.49 & 3.06 & - & 0.33 & 1.17 & 0.24 & 0.30 & 2.58 & 0.38 & 1.20 & 1.53 \\
\hline Globulol (1609) & - & 0.33 & - & - & - & - & - & 0.42 & 1.06 & - & 0.24 & - \\
\hline T-Cadinol (1639) & - & & - & - & - & - & - & - & - & - & 0.61 & - \\
\hline Eudesm-7(11)-en-4- ol (1644) & - & 0.59 & - & - & 0.43 & - & - & 1.79 & - & - & 0.16 & - \\
\hline T-Muurolol (1660) & - & - & - & - & 0.85 & 0.49 & - & 0.15 & - & - & - & - \\
\hline$\alpha$-Cadinol (1673) & - & - & - & 0.39 & 0.47 & 0.71 & - & 0.11 & 0.93 & - & - & 0.21 \\
\hline Eudesm-4(14)-en-11-ol (1676) & - & 0.87 & - & - & - & - & - & - & - & - & 1.14 & - \\
\hline Viridiflorol (1691) & - & - & 0.36 & - & - & - & - & - & 1.17 & - & - & - \\
\hline $\begin{array}{l}\text { 6-Isopropenyl-4,8a- dimethyl-1,2,3,5,6,7,8,8a- } \\
\text { octahydro-2- naphthalenol(1690) }\end{array}$ & & - & 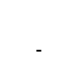 & 0.64 & 0.68 & 0.62 & - & 0.59 & 1.98 & - & 0.43 & - \\
\hline Spathulenol (1705) & 1.35 & 0.06 & . & 3.80 & 5.49 & 2.43 & 1.14 & 1.15 & - & 0.53 & 1.74 & 0.50 \\
\hline Alloaromadendrene oxide(1788) & - & 2.34 & - & - & - & - & - & - & 1.08 & - & 2.15 & 1.18 \\
\hline Other oxygenated sesquiterpenes derivatives & . & 0.49 & - & - & 0.57 & - & - & 0.23 & - & - & 0.74 & - \\
\hline Sub-total & 13.64 & 12.47 & 16.74 & 4.83 & 9.54 & 5.42 & 1.38 & 4.98 & 8.80 & 4.01 & 14.05 & 4.10 \\
\hline
\end{tabular}

Miscellaneous compounds

trans-3,6-diethyl-3,6-dimethyl- tricyclo[3.1.0.0(2,

6,7-dimethyl-1,2,3,5,8,8a- hexahydronaphthalene

(1604)

Andrographolide (1612)

$\begin{array}{ccc}- & - & -\end{array}$

$0.44 \quad 0.15$

0.07

0.39

Sub-total

$0.44 \quad 0.15$

$\begin{array}{cccccccc}- & - & - & - & - & - & 0.36 & - \\ \mathbf{0 . 0 7} & \mathbf{0 . 3 9} & \mathbf{0 . 0 0} & \mathbf{0 . 0 0} & \mathbf{0 . 0 0} & \mathbf{0 . 0 0} & \mathbf{0 . 7 7} & \mathbf{0 . 0 0}\end{array}$

Note: KI*: Kovat's indices on BPX-5 column compared with Liu et al. (2011). Unidentified components which are less than 0.5\% are not reported. KF: Kuching variety (fruit); KFS: Kuching variety (fruit stalk); KSB Note: KI*: Kovat's indices on BPX-5 column compared with Liu et al. (2011). Unidentified components which are less than 0.5\% are not reported. KF: Kuching variety (fruit); KFS: Kuching variety (fruit stakk); KSB
variety (stem bark); EF: Semongok Emas variety (fruit); EFS: Semongok Emas variety (fruit stalk); ESB: Semongok Emas variety (stem bark); PF: Semongok Perak variety (fruit); PFS: Semongok Perak variety (fruit st: variety (stem bark); EF: Semongok Emas variety (fruit); EFS: Semongok Emas variety (fruit stalk); ESB: Semongok Emas variety (stem bark); PF: Semongok Perak variety (fruit); PFS: Semongok Perak variety (fruit ste
Semongok Perak variety (stem bark); WF: Semongok Wan variety (fruit); WFS: Semongok Wan variety (fruit stalk); WSB: Semongok Wan variety (stem 



\section{CONCLUSIONS}

The oil constituents of four $P$. nigrum varieties showed the predominance of sesquiterpenes, except for Semongok Emas and Semongok Wan fruit oils. Hydrocarbon sesquiterpenes were identified as the major components in all oil samples studied. The chemometric analysis based on the GC-MS data of oil illustrated that the oil compositions among the fruit, fruit stalk and stem bark in four different $P$. nigrum varieties are quite similar. A considerable level of similarity between the varieties was also distinguished. Further study on other plant parts of various $P$. nigrum varieties may provide valuable information for current chemotaxonomic investigation based on essential oil composition.

\section{ACKNOWLEDGEMENTS}

The authors are grateful to the Universiti Malaysia Sarawak (UNIMAS) for financial support to Irna Syairina Sahari via Postgraduate Fellowship Scheme. We also like to acknowledge the officials of Tarat Agricultural Seeding Production Centre for providing $P$. nigrum samples.

\section{REFERENCES}

Adams, R.P. (1995). Identification of Essential Oil Components by Gas Chromatography/Mass Spectrometry. Carol Stream, IL., USA: Allured, 804pp.

Amma, S.P., Nybe, E.V., Sujatha, V.S. \& Prabhakaran, P.V. (2001). Survey, evaluation and identification of black pepper cultivars. Journal of Tropical Agriculture, 39: 9-12.

Barceloux, D.G. (2009). Pepper and capsaicin (Capsicum and Piper Species). Disease-aMonth, 55(6): 380-390.

Det, P.A. (2008). Other pepper varieties cultivated in Sarawak. http://www.ctahr.hawaii.edu/sustainas/extn _pub/veggie\%20pubs/Pepper\%20EFS05.pdf

Janic, J. (2007). Plant Breeding Reviews, New Jersey: John Wiley and Sons, 232pp.
Liu, J., Bi, Y., Luo, R. and Wu, X. (2011). Simultaneous UFLC-ESI-MS/MS determination of piperine and piperlonguminine in rat plasma after oral administration of alkaloids from Piper longum L.: Application to pharmacokinetic studies in rats. Journal of Chromatography, B 879(27): 2885-2890.

Mammootty, K.P., Abraham, K. \& Vijayaraghavan, R. (2008). Short communication: Screening black pepper (Piper nigrum L.) varieties/cultivars against Phytophthora disease in the nursery. Journal of Tropical Agriculture, 46(1-2): 70-72.

Martins, A.P., Salgueiro, L., Vila, R., Tomi, F., Canigueral, S., Casanova, J., Cunha, A.P.D. \& Adzet, T. (1998). Essential oils from four Piper species. Phytochemistry, 49(7): 20192023.

Menon, A.N., Padmakumari, K.P. \& Jayalekshmy, A. (2003). Essential oil composition of four major cultivars of black pepper (Piper nigrum L.) III. Journal of Essential Oil Research, 15(3): 155-157.

Menon, A.N. \& Padmakumari, K.P. (2005a). Essential oil composition of four major cultivars of black pepper (Piper nigrum L.) - IV. Journal of Essential Oil Research, 17(2): 206-208.

Menon, A.N. \& Padmakumari, K.P. (2005b). Studies on essential oil composition of cultivars of black pepper (Piper nigrum L.) - V. Journal of Essential Oil Research, 17(2): 153-155.

Pino, J.A., Aguero, J. \& Fuentes, V. (2003). Chemical composition of the aerial parts of Piper nigrum L. from Cuba. Journal of Essential Oil Research, 15: 209-210.

Ravindran, P.N. \& Kallupurackal, J.A. (2000). Black pepper. In: Peter, K.V. (Ed.), Handbook of Herbs and Spices (pp $62-$ 110). Cambridge, England: Woodhead Publishing Limited.

Sasidharan, I. \& Menon, A.N. (2010). Comparative chemical composition and antimicrobial activity of berry and leaf essential oils of Piper nigrum L. International Journal of Biological \& Medical Research, 1(4): 215-218. 
Tchoumbougnang, F., Jazet Dongmo Pierre, M., Sameza Modeste, L., Fombotioh, N., Wouatsa Nangue Arlette, V., Amvam Zollo Paul, H. \& Menut, C. (2009). Comparative essential oils composition and insecticidal effect of different tissues of Piper capense L., Piper guineense Schum. et Thonn., Piper nigrum L. and Piper umbellatum L. grown in Cameroon. African Journal of Biotechnology, 8(3): 424-431. 